\title{
Impact of pharmacological Interventions on fertility outcomes in women with polycystic ovary syndrome: a systematic review and meta-analysis of randomised controlled trials
}

\author{
MOHAMMED ABDALLA ${ }^{1}$, Najeeb Shah ${ }^{1}$, Harshal Deshmukh², Amirhossein Sahebkar ${ }^{3}$, \\ Linda Östlundh ${ }^{4}$, Rami Al-Rifai ${ }^{4}$, Stephen Atkin ${ }^{5}$, and Thozhukat Sathyapalan ${ }^{1}$ \\ ${ }^{1}$ University of Hull \\ ${ }^{2}$ Hull University Teaching Hospitals NHS Trust \\ ${ }^{3}$ Mashhad University of Medical Sciences \\ ${ }^{4}$ United Arab Emirates University \\ ${ }^{5}$ Royal College of Surgeons in Ireland and Medical University of Bahrain
}

September 25, 2021

\begin{abstract}
Background: Polycystic ovary syndrome (PCOS) is an endocrine condition associated with sub-fertility, infertility and poor reproductive outcomes. Objectives: To review the effectiveness of different pharmacological interventions on fertility outcomes in women with PCOS. Search Strategy: We searched PubMed, MEDLINE, Scopus, Embase, Cochrane Library, Web of Science in April 2020 and updated the search in PubMed March 2021. Selection Criteria: Two independent reviewers selected studies, and only randomised controlled trials (RCTs) were included. Data Collection and Analysis: Thirty-four RCTs that met the eligibility criteria were used to calculate odds ratios (OR) and the 95\% confidence interval (95\% CI) using the random effect model. Main Results: There was a significant increase in pregnancy rate with follicular stimulating hormone (FSH) vs clomiphene citrate (CC)+ metformin (Odd Ratio(OR):4.08; 95\%CI:1.12-14.83, $\mathrm{I}^{2}=79 \%$ ), Letrozole vs CC (OR: 1.58; 95\%CI: 1.34-1.86, $\mathrm{I}^{2}=0 \%$ ), metformin vs placebo(OR: $\left.3.00 ; 95 \% \mathrm{CI}: 1.95-4.59, \mathrm{I}^{2}=0 \%\right)$ and with CC+ metformin vs CC (OR: 1.48 ; 95\% CI: $\left.1.02-2.16, \mathrm{I}^{2}=39 \%\right)$. There were significant increases in ovulation rate with CC+ metformin vs FSH (OR: 0.09; $95 \%$ CI: 0.02-0.37, $\left.\mathrm{I}^{2}=75 \%\right), \mathrm{CC}+$ metformin vs CC (OR: 2.04; 95\%CI: $\left.1.35-3.08, \mathrm{I}^{2}=63 \%\right)$ and with Letrozole vs CC (OR: 1.60 ; 95\% CI: $1.02-2.52, \mathrm{I}^{2}=88 \%$ ). A significant increase in live birth with Letrozole vs CC (OR: $1.63 ; 95 \%$ CI: $1.21-2.21, \mathrm{I}^{2}=0 \%$ ) was observed. Conclusions: CC, letrozole alone or either added to metformin, were associated with a significant increase in the pregnancy rate, ovulation rate, and live birth rate in women with PCOS. Funding: No fund for the review.
\end{abstract}

\section{Hosted file}

Fertility outcome.edited.docx available at https://authorea.com/users/437483/articles/539087impact-of-pharmacological-interventions-on-fertility-outcomes-in-women-with-polycysticovary-syndrome-a-systematic-review-and-meta-analysis-of-randomised-controlled-trials 